\title{
The slow-neutron capture process in low-metallicity asymptotic giant branch stars
}

\author{
Amanda I. Karakas ${ }^{1}$, Maria Lugaro ${ }^{2}$, and Simon W. Campbell ${ }^{3}$ \\ ${ }^{1}$ Mount Stromlo Observatory, Australian National University, Weston Creek ACT 2611, \\ Australia \\ email: akarakas@mso.anu.edu.au \\ ${ }^{2}$ Centre for Stellar and Planetary Astrophysics, Monash University, Clayton 3800, Victoria, \\ Australia \\ email: Maria.Lugaro@sci.monash.edu.au \\ ${ }^{3}$ Astronomy and Astrophysics Group, Department Fisica i Enginyeria Nuclear, Universitat \\ Politecnica de Catalunya, Barcelona, Spain \\ email: simwcampbell@gmail.com
}

\begin{abstract}
Elements heavier than iron are produced in asymptotic giant branch (AGB) stars via the slow neutron capture process ( $s$ process). Recent observations of $s$-process-enriched Carbon Enhanced Metal-Poor (CEMP) stars have provided an unprecedented wealth of observational constraints on the operation of the $s$-process in low-metallicity AGB stars. We present new preliminary full network calculations of low-metallicity AGB stars, including a comparison to the composition of a few $s$-process rich CEMP stars. We also discuss the possibility of using halo planetary nebulae as further probes of low-metallicity AGB nucleosynthesis.
\end{abstract}

Keywords. stars: abundances - stars: AGB and post-AGB

\section{Introduction}

Asymptotic giant branch (AGB) stars play a crucial role in the chemical evolution of elements heavier than iron. The operation of the $s$-process in AGB stars synthesized up to half of all elements heavier than $\mathrm{Fe}$ in the solar system, with most of the $\mathrm{Pb}$ on Earth coming from low-metallicity AGB stars. Heavy elements, such as Zr, Ba, and Pb, are mixed from the deep interior to the stellar surface by recurrent mixing episodes. Strong stellar winds then expel this enriched matter into the interstellar medium, thus contributing to the chemical evolution of galaxies and stellar systems.

Detailed neutron-capture abundance information is now known for many extremely metal-poor field and globular cluster stars in the Galactic halo. Many of the metalpoor field halo stars are also greatly enhanced in carbon, the Carbon Enhanced Metal Poor (CEMP) stars, along with elements that can be produced by the slow neutron capture process (the $s$ process) and/or rapid neutron capture process (the $r$ process; see reviews by Beers \& Christlieb 2005 and Sneden, Cowan, \& Gallino 2008). That many of the CEMP stars with $s$ process enhancements (hereafter CEMP- $s$ stars) are also in long-period binary systems (Lucatello et al. 2005) has led to the hypothesis that these stars obtained their carbon and heavy element abundances via mass transfer from a now extinct asymptotic giant branch (AGB) companion. Comparison between CEMP- $s$ abundance data with theoretical stellar models of AGB stars has further supported this hypothesis (e.g., Ivans et al. 2005).

In these proceedings, we report on new preliminary theoretical predictions of $s$ process nucleosynthesis from low-mass, low-metallicity AGB stars. These models are compared to the composition of a couple of CEMP stars with enhancements of neutron-capture 
elements. We also discuss the potential of using halo planetary nebulae as probes of heavy element nucleosynthesis in low-mass AGB stars.

\section{Low-metallicity Asymptotic Giant Branch Stars}

The numerical method we use has previously been described in detail (e.g., Karakas \& Lattanzio 2007). We first compute the stellar structure using the Mt Stromlo Stellar Structure code, where each model is evolved from the zero-age main sequence to near the tip of the TP-AGB. The structure is then used as input into a post-processing nucleosynthesis code where we obtain abundances for many more species (291) than are included in the structure model (6 species). The stellar models computed for this study are of mass $1.5 M_{\odot}$ and $2 M_{\odot}$, with $Z=0.0001([\mathrm{Fe} / \mathrm{H}] \approx-2.3)$.

For this study we extended the nuclear network to include all elements from iron to bismuth. We include 291 species from protons to sulfur, and iron through to Bi. For reaction rates, we have used the JINA REACLIB library $\dagger$, except for the ${ }^{13} \mathrm{C}(\alpha, n){ }^{16} \mathrm{O}$ reaction which we have updated to the Heil et al. (2008) rate, and the ${ }^{22} \mathrm{Ne}+\alpha$ rates, which are from Karakas et al. (2006).

In each model we include a partial mixing zone of $0.002 M_{\odot}$ at the deepest extent of each third dredge up episode. In this zone protons penetrate into the top layers of the Heintershell, where they are captured by the abundant ${ }^{12} \mathrm{C}$ to form a ${ }^{13} \mathrm{C}$ (and ${ }^{14} \mathrm{~N}$ ) pocket. It is in the ${ }^{13} \mathrm{C}$ pocket that the reaction ${ }^{13} \mathrm{C}(\alpha, n){ }^{16} \mathrm{O}$ operates to release free neutrons that are captured by iron-seed nuclei. During thermal pulses the ${ }^{22} \mathrm{Ne}(\alpha, n)^{25} \mathrm{Mg}$ neutron source is also activated. For further details we refer the reader to Karakas et al. (2009).

The initial composition used in most of the calculations are scaled solar, where we use the solar abundances from Anders \& Grevesse (1989). We also computed models assuming initial $[\mathrm{Ba} / \mathrm{Fe}]=1.5$ and $[\mathrm{Eu} / \mathrm{Fe}]=2.0$ to simulate a primordial rapid neutron capture $(r$ process $)$ enrichment. We use the standard spectroscopic notation $[\mathrm{X} / \mathrm{Fe}]=$ $\log (\mathrm{X} / \mathrm{Fe})-\log (\mathrm{X} / \mathrm{Fe}) \odot$.

\section{Comparison to CEMPS}

In Figs. 1 and 2 we show the predicted $[\mathrm{X} / \mathrm{Fe}]$ abundances versus atomic mass for the $1.5 M_{\odot}$ and $2 M_{\odot}, Z=0.0001$ models, with initial $\mathrm{Ba}$ and $\mathrm{Eu}$ abundances as indicated in the figure caption. In Fig. 1 the mean abundances for the CEMP star HD 196944 from the studies of Van eck et al. (2001) and Aoki et al. (2002) are shown. Overlaid on Fig. 2 are the derived abundance of the CEMP star CS 29497-030, with data from Ivans et al. (2005). Observational errors are not included in either figure but are on the order of 0.1-0.3 dex depending on the element, see the references for further details.

In these figures we are not trying to provide a perfect match to the observational data, but to show preliminary examples of the model predictions. In Fig. 1 the general pattern of the observed distribution is matched by the model but all of the observed data points lie at the lower end of the predicted values. This indicates that there was substantial dilution of the AGB wind with the envelope of the accretor star. This is plausible as HD 196944 is a giant star, thus it has an extended convective envelope. As discussed in Ivans et al. (2005), CS 29497-030 requires an AGB model pre-enriched with $r$-process material to provide the best match to the observed data.

$\dagger$ http://groups.nscl.msu.edu/jina/reaclib/db/ 


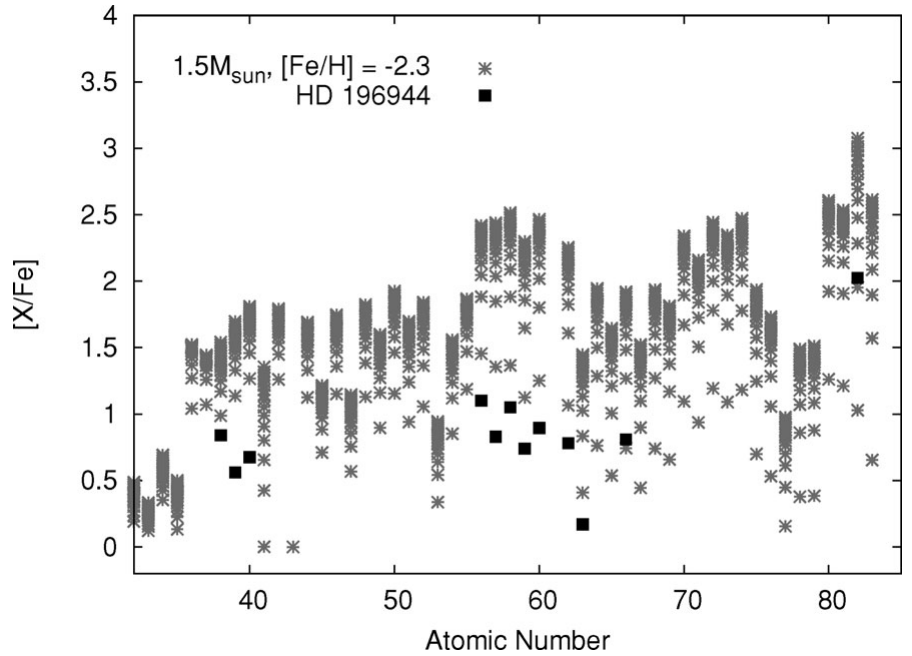

Figure 1. Predicted temporal evolution of $[\mathrm{X} / \mathrm{Fe}]$ versus atomic number during the thermally-pulsing AGB phase. The $[\mathrm{X} / \mathrm{Fe}]$ values evolve from lower to higher values during the TP-AGB phase. Shown are results for the $1.5 M_{\odot}, Z=0.0001$ model with initial $[\mathrm{Ba} / \mathrm{Fe}]=0.0$ and $[\mathrm{Eu} / \mathrm{Fe}]$ $=0.0$. Overlaid are abundances derived for the CEMP star HD 196944, taking the mean of abundances from Aoki et al. (2002) and Van Eck et al. (2001).

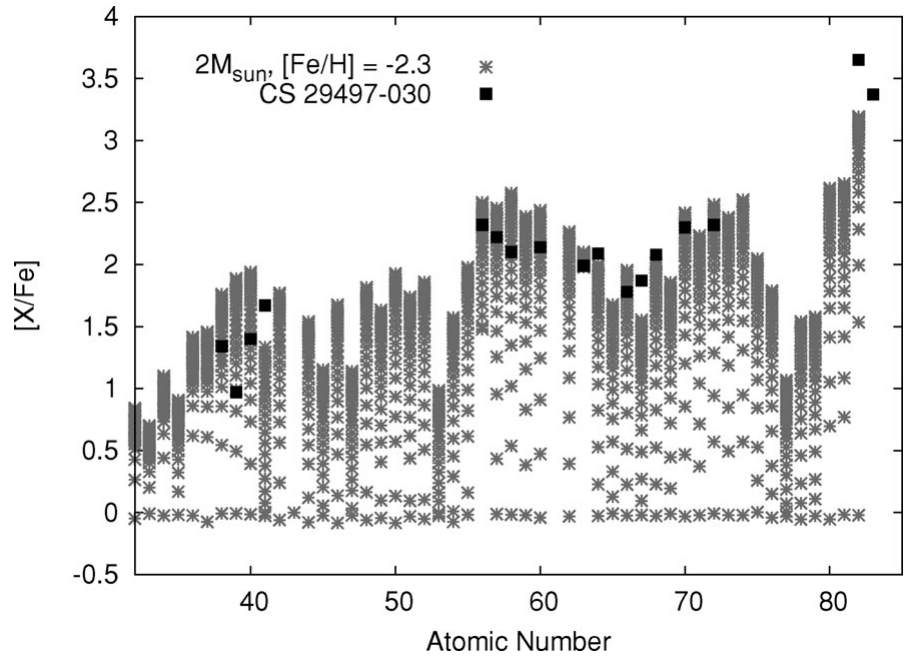

Figure 2. Predicted temporal evolution of $[\mathrm{X} / \mathrm{Fe}]$ versus atomic number during the thermally-pulsing AGB phase. Shown are results for the $2 M_{\odot}, Z=0.0001$ model with initial $[\mathrm{Ba} / \mathrm{Fe}]=$ 1.5 and $[\mathrm{Eu} / \mathrm{Fe}]=2.0$. Overlaid are abundances derived for the CEMP star CS 29497-030 from Ivans et al. (2005).

In a future study we plan to compare our model predictions to larger numbers of CEMP- $s$ and CEMP $-r+s$ stars, covering a larger range in initial progenitor AGB mass and metallicity.

\section{Halo planetary nebulae}

The determination of heavy element abundances from planetary nebula (PN) spectra provides an exciting opportunity to study nucleosynthesis that occurred previously in the progenitor asymptotic giant branch (AGB) star. Abundances derived from PN 
spectra provide a complimentary data set to the abundances derived from the spectra of cool evolved stars. Observations have revealed that some PNe are enriched in the heavy elements that can be produced by the $s$-process including $\mathrm{Ge}, \mathrm{Se}, \mathrm{Kr}$, Xe, and Ba (e.g., Sterling \& Dinerstein 2008). Sterling \& Dinerstein (2008) obtained Se and Kr abundances for 120 Galactic PNe, and investigated trends between $s$-process enrichments and PN morphology and other nebular and stellar characteristics.

All of the PNe considered by Sterling \& Dinerstein (2008) had metallicities appropriate for the Galactic disk. There are a few PNe that have been identified in the Galactic halo. Neutron capture-element information from these objects could be used as a complimentary data set to the CEMP stars, allowing these low-metallicity PNe to be used as tracers of the formation of the Galaxy at the earliest times, and to constrain the evolution and nucleosynthesis of low-metallicity AGB stars.

In Karakas \& Lugaro (2009) we compared $s$-process predictions from low-mass $(1.25$ and $\left.2 M_{\odot}\right)$ AGB stars with $[\mathrm{Fe} / \mathrm{H}]=-2.3$ to Se and $\mathrm{Kr}$ abundances found in Galactic PNe from Sterling \& Dinerstein (2008). Predictions for elements between Fe and Rb were also given. Oxygen was used as a proxy for metallicity, i.e., [X/O] instead of Fe, to be consistent with observations. Taking $\mathrm{Zn}$ as an example, the predicted abundance of $[\mathrm{Zn} / \mathrm{O}]$ is sub-solar, with $[\mathrm{Zn} / \mathrm{O}] \sim-1$ (starting at $[\mathrm{Zn} / \mathrm{O}]=-0.4$, scaled solar for $\mathrm{Zn}$ ). At these low metallicities, the dredge-up of oxygen during the AGB substantially increases the surface composition, from $[\mathrm{O} / \mathrm{Fe}]=0.4$ (typical of the halo) to $[\mathrm{O} / \mathrm{Fe}] \approx 1.0$. In all cases, $\mathrm{Zn}$ is actually produced by the $s$-process but in smaller quantities to oxygen, hence the $[\mathrm{X} / \mathrm{O}]$ ratios do not reflect the degree of production. For example, the final $[\mathrm{Zn} / \mathrm{Fe}]=0.45$. Note that the iron abundance remains essentially unchanged.

Our results indicate that at these low metallicities, oxygen is no longer a suitable proxy for the initial metallicity of the progenitor star. Argon, if available, would be a more suitable proxy, as its abundance remains unaltered by AGB nucleosynthesis. For PNe neutron-capture elements to be useful, the issue of a suitable metallicity proxy would need to be resolved.

\section{Summary}

We have computed new full network predictions from low-mass AGB stars of low metallicity. These predictions are to be compared to the derived composition of carbonenhanced metal-poor stars of similar metallicity as the models. We also discuss the possibility of using halo planetary nebulae as further probes of low-metallicity AGB nucleosynthesis. Such comparisons allow the physics and nucleosynthesis of AGB models to be constrained, and provide insight into the formation of the Galaxy at the earliest times.

\section{References}

Aoki, W., et al. 2002, ApJ, 580, p. 1149

Beers, T. C. \& Christlieb, N. 2005, ARA\&A, 43, p. 531

Heil, M. et al. 2008, Physical Review C, 78, 025803

Ivans, I. I., Sneden, C., Gallino, R., Cowan, J. J., \& Preston, G. W. 2005, ApJ, 627, L145

Karakas, A. I. \& Lattanzio, J. C. 2007, PASA, 24, p. 103

Karakas, A. I. \& Lugaro, M. 2009, PASA, accepted.

Karakas, A. I., et al. 2009, ApJ, 690, p. 1130

Lucatello, S. et al. 2005, ApJ, 625, p. 824

Sneden, C., Cowan, J. J., \& Gallino, R. 2008, ARA\&A, 46, p. 241

Sterling, N. C. \& Dinerstein, H. L. 2008, ApJS, 174, 158

Van Eck, S., Goriely, S., Jorissen, A., \& Plez, B. 2001, Nature, 412, p. 793 